\title{
Effect of Nettle Root Extract on Histometrical Parameters of Cerebral and Cerebellar Cortices in Rat Following Administration of Testosterone
}

Sajad Sahab Negah", ${ }^{1,}$ Zabihollah Khaksar ${ }^{2}$, Shahin Mohammad Sadeghi ${ }^{3}$, Naeem Erfanimajd ${ }^{4}$, Sayed Mostafa Modarres Mousavi', Hadi Aligholi', ${ }^{1,}$, Masoud Adibmoradi', Hamid Reza Moradi ${ }^{6 *}$

${ }^{1}$ Shefa Neuroscience Research Center, Khatam Alanbia Hospital, Tehran, Iran.

${ }^{2}$ Histology and Embryology Group, Department of Basic Science, Faculty of Veterinary Medicine, Shiraz University, Shiraz, Iran.

${ }^{3}$ Department of Plastic and Reconstructive Surgery, Shahid Beheshti University of Medical Sciences, Tehran, Iran.

${ }^{4}$ Department of Basic Sciences, Faculty of Veterinary Medicine, Shahid Chamran University of Ahvaz, Ahvaz, Iran.

${ }^{5}$ Neuroscience Group, School of Advanced Technologies in Medicine, Tehran University of Medical Sciences, Tehran, Iran.

${ }^{6}$ Department of Basic Sciences, Faculty of Veterinary Medicine, Tehran University, Tehran, Iran.

\section{ABSTRACT}

Introduction: Testosterone is a steroid hormone secreted by gonads and adrenal glands. High dose of testosterone leads to decrease neurogenesis and induce apoptosis in vitro. To modulate testosterone effect, plants contain anti-androgenic properties can be useful. Urtica dioica, often called common nettle, contains anti-androgen compounds. Furthermore, the phytoestrogens effects of nettle have been evaluated. In this study, the effect of nettle root extract on histological structures of the cerebral and cerebellar cortices of rats was evaluated after testosterone injection. Materialsand Methods: Twenty healthy adult male Wistar rats were divided randomly into 4 groups: control group (received ordinary feed without any treatment), testosterone group (received $10 \mathrm{mg} / \mathrm{kg}$ testosterone subcutaneously), nettle root extract group (received $50 \mathrm{mg} / \mathrm{kg}$ nettle root extract orally), nettle + testosterone group (received $50 \mathrm{mg} / \mathrm{kg}$ orally nettle root extract and $10 \mathrm{mg} / \mathrm{kg}$ testosterone). After 6 weeks, the brains were stained by hematoxylin and eosin. The number of neuronal cell body, nuclear diameter of neurons, diameter of Purkinje cells, and diameter of cerebellar layers were measured. Results: There were no significant differences in the mean number of neuronal cell body in cerebral cortex, diameter of Purkinje cells in cerebellum, and thickness of cerebellar layers among different groups. The nuclear diameter of neurons in inner granular layer of cerebrum in the testosterone group significantly increased compared to the nettle and nettle + testosterone groups. Conclusion: Nettle plant can be considered as a testosterone modulator. To determine more precise effect of nettle on the brain, further studies are needed.

\section{Key words:}

1. Brain

2. Rats

3. Urtica dioica

4. Testosterone

* Corresponding Author: Hamid Reza Moradi

E-mail: hmoradi20@yahoo.com 


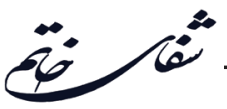

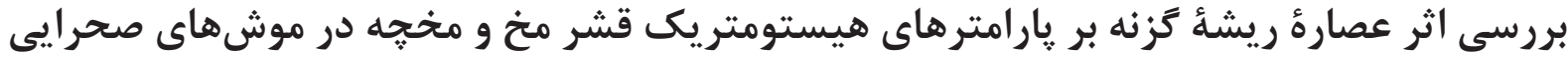 متعاقب تجويز تستوسترون هيستونئ}

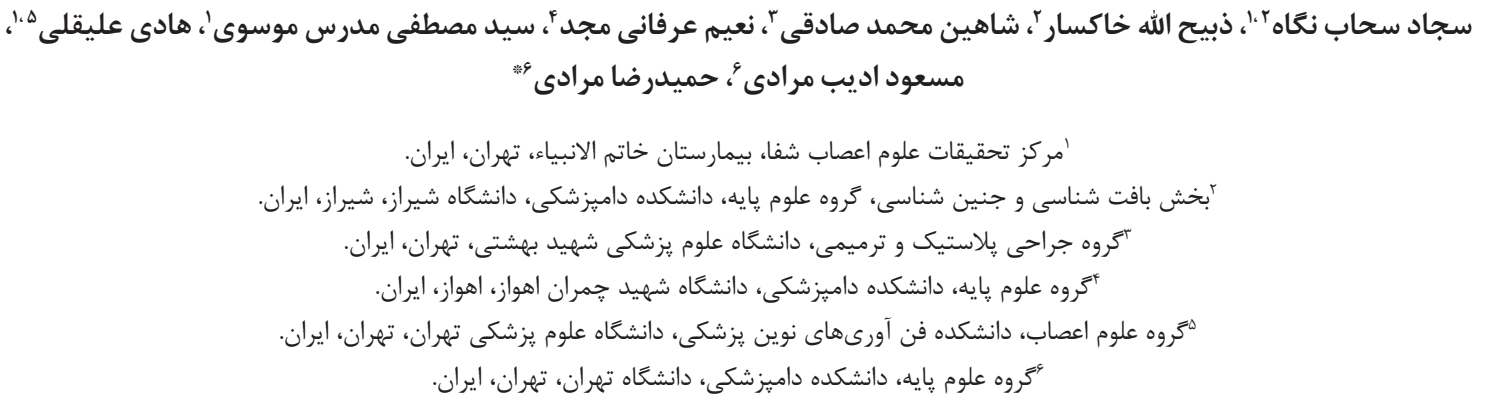

كليد وازهها:

I

r. r. موشهاى صحرايى

r. او اورتيكا ديوئيكا

t

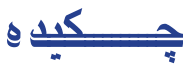

مقدمه: تستوسترون يك هورمون استروئيدى است كه توسط كنادها و غدد آدرنال ترشح مىشود. دوز

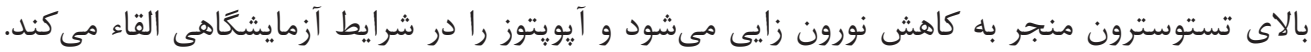

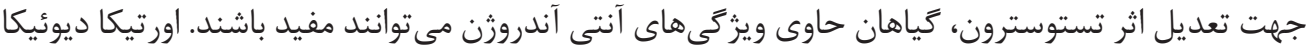

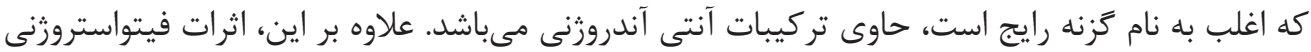

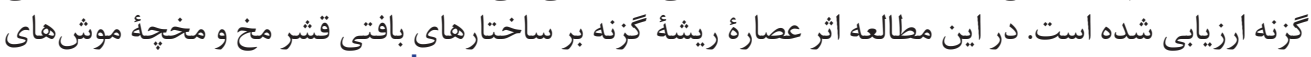

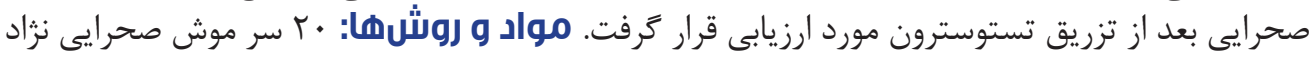

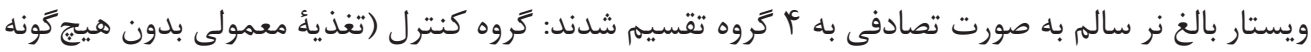

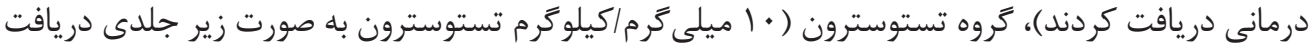

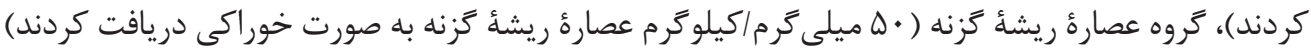

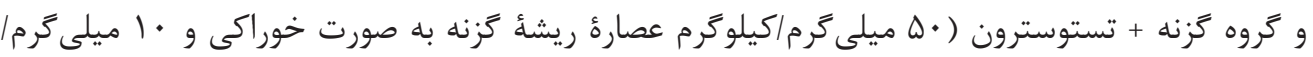

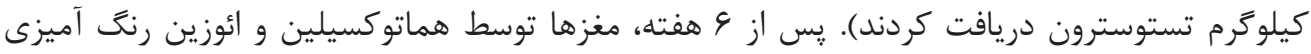

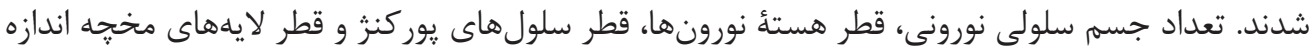

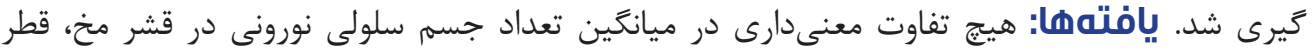

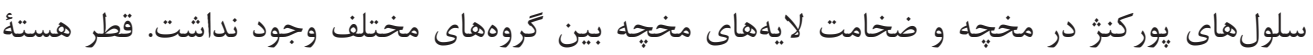

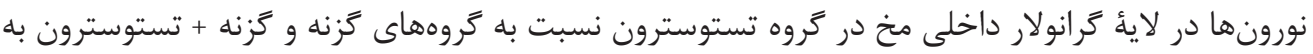

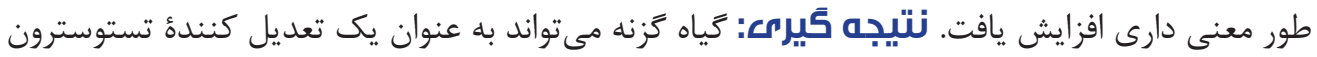

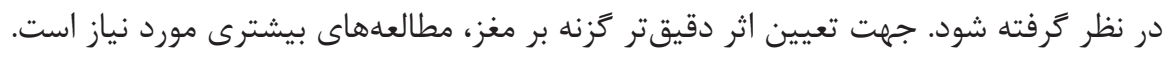

" نويسنده مسئول: حميدرضا مرادى : آدرس الكترونيكى: hmoradi20@yahoo.com 


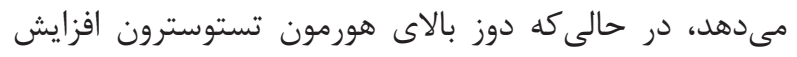
حجم انفار كتوس را به همراه دارد (1) (1). با توجه به اينكه دوز بالاى تستوسترون منجر به ايجاد تغييرات

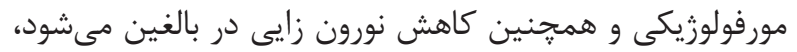

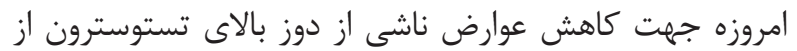

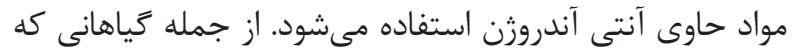

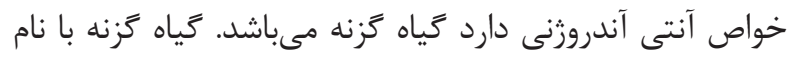

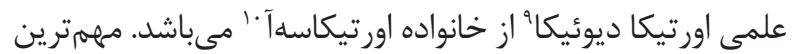

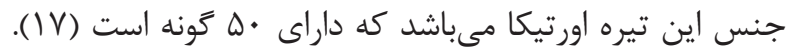

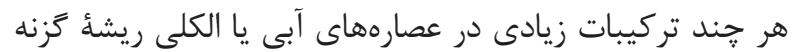

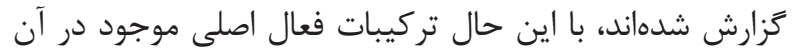

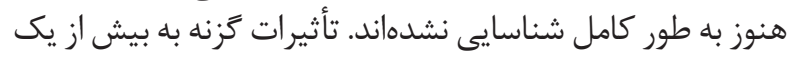

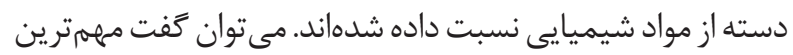

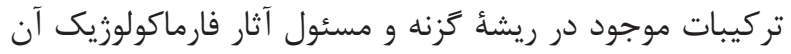

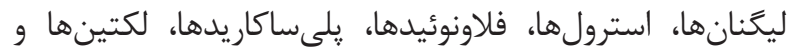

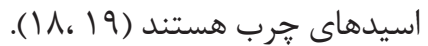

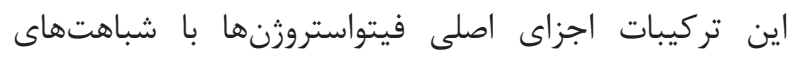

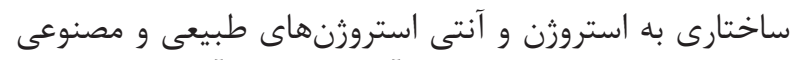

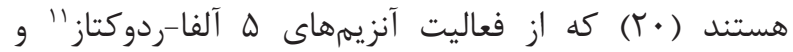

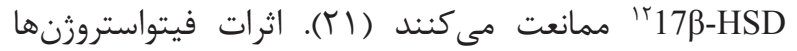
بر سيستم عصبى مركزى كمتر مورد توجه قرار گرفته است. ايزوفلاونهاى موجود در فيتواستروزن ها موجب مرئ افزايش سطوح

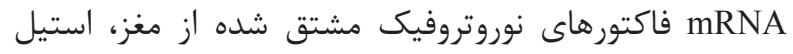

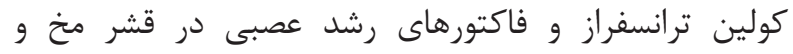

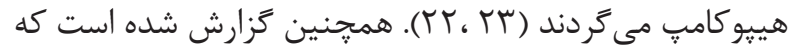

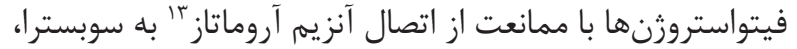
با آندروزن رقابت مى كنند (YY)

در مطالعهاى جهت درمان هيير يلازى يروستات ناشى از دوز

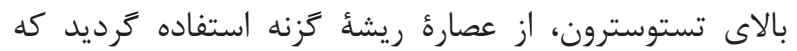

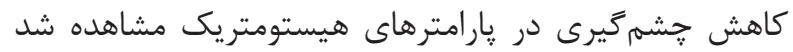

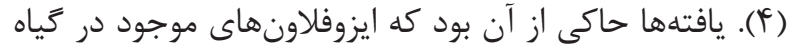

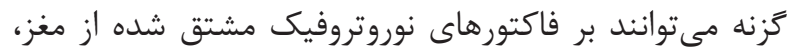

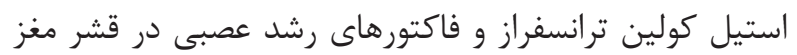

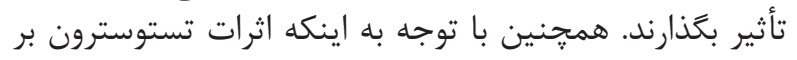

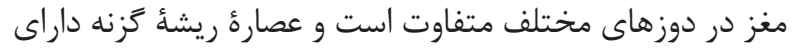

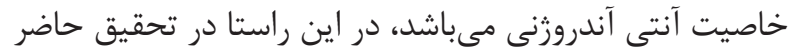

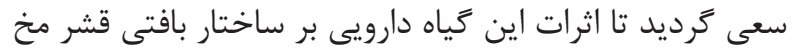

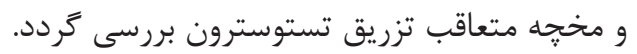

مواد و روشها - - ماد

تستوسترون از شركت نيل مصر، قطرء خوراكى اورتيدين (عصاره

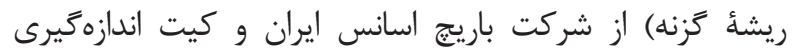

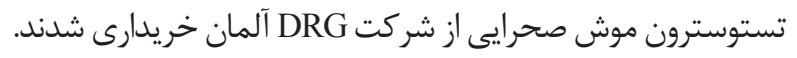

${ }^{1}$ Hypertension

${ }^{2}$ Atherosclerosis

${ }^{3}$ Hepatic neoplasms

${ }^{4}$ Carcinoma

${ }^{5}$ Tendon damage

${ }^{6}$ Hyperplasia

${ }^{7}$ Neurogenesis
مقدمه - - مق

تستوسترون هورمونى است كه توسط غدد جنسى و آدرنال توليد مى

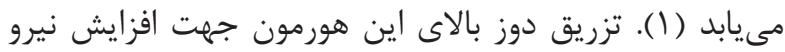

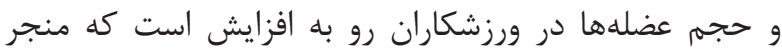

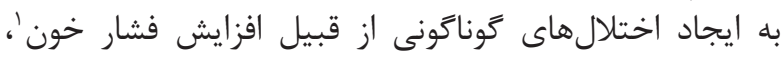

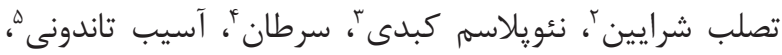

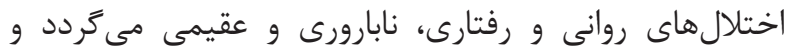

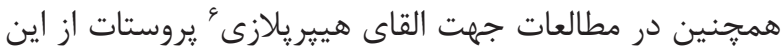

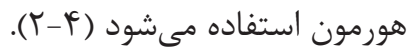

هورمونهاى جنسى به خصوص تستوسترون در زمان بلوغ

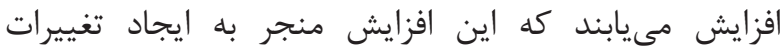

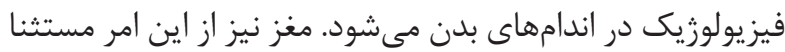

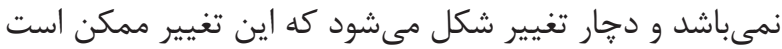

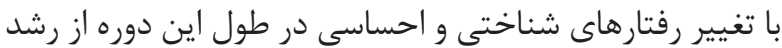

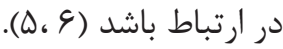

مطالعههاى انجام شده در انسان و مدل هاى آزمايشگاهى نشان

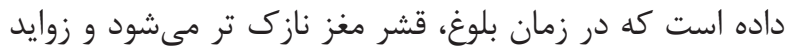

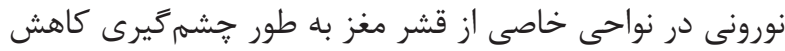

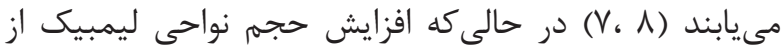

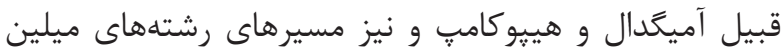

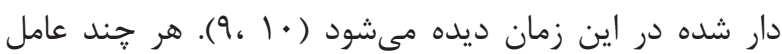

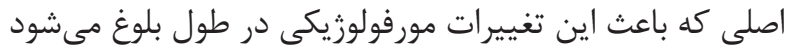

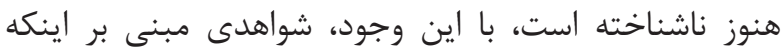

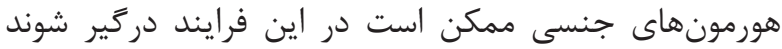

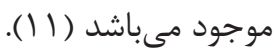

علاوه بر تغييرات مورفولوزيكى ممكن است تغيير در سطح

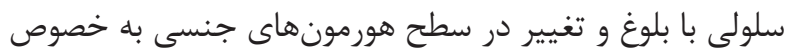

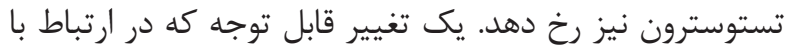

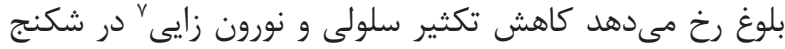

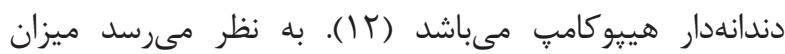

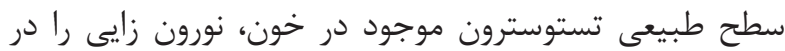

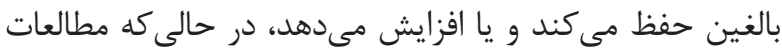

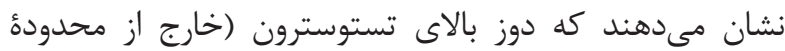

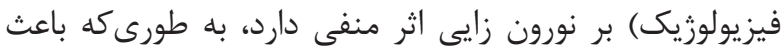

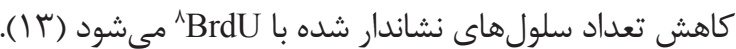
مطالعات در شرايط In vitro نيز گوياى اين امر است به طورى كه

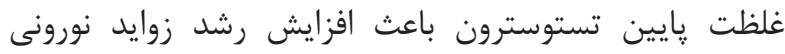

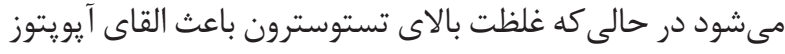

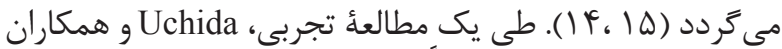

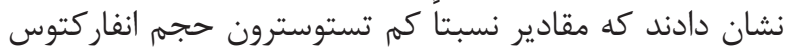

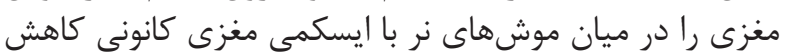

\footnotetext{
${ }^{8}$ Bromodeoxyuridine

${ }^{9}$ Urtica dioica

${ }^{10}$ Urticaceae

${ }^{11} 5 \alpha$ Reductase

${ }^{12}$ 17Beta Hydroxysteroid Dehydrogenase

${ }^{13}$ Aromatase enzyme
} 
همجنين قطر سلولهاى يور كنز اندازه گَيرى شد. ميانكين تعداد

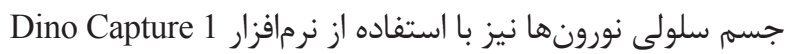

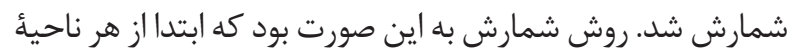

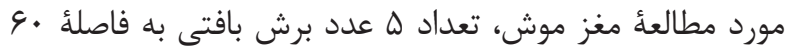

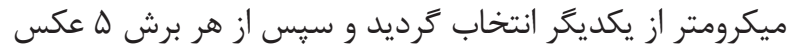

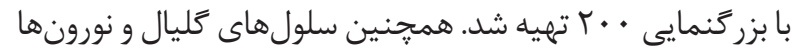

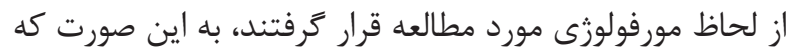

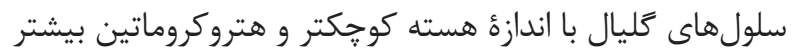
نسبت به نورونها تشخيص داده شداند.

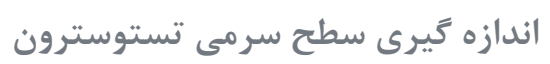

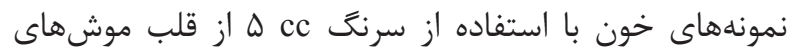

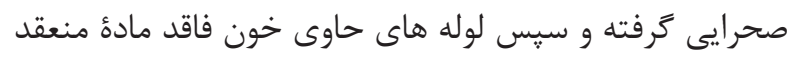

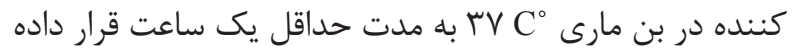

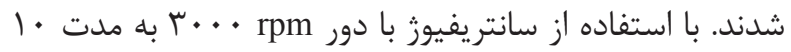

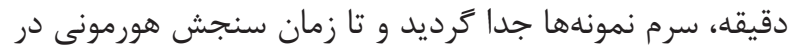

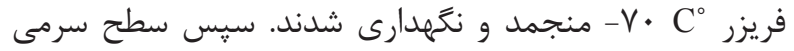

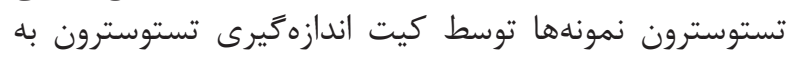
روش الايزا اندازه كيرى شدند.

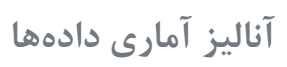

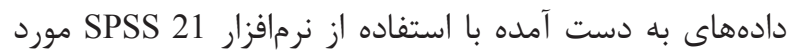

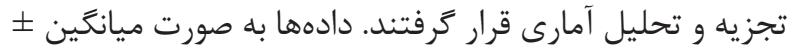

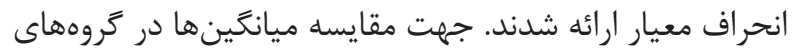

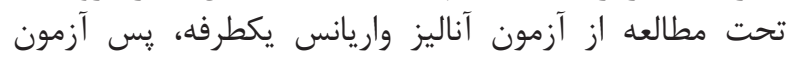

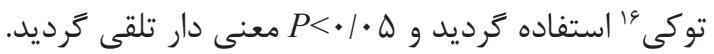

بافتهها

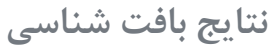

نتايج بافت شناسى حاصل از اين تحقيق نشان داد كه سلولهاى

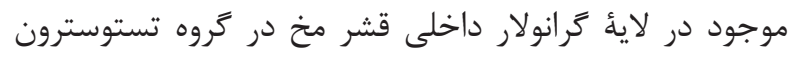

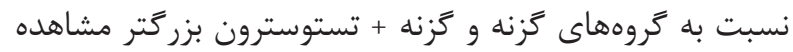

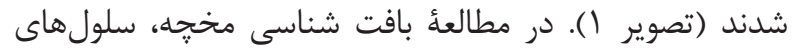

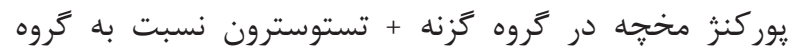
كنترل بزر كتر ديده شدند (تصوير r) ).

بررسى هاى هيستومترى قطر هستأ نورونها، در بين خروهوهاى

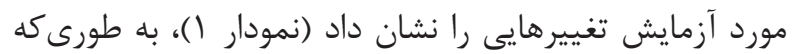

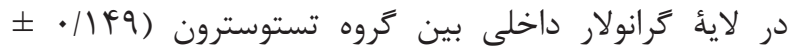

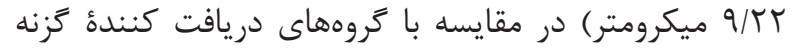

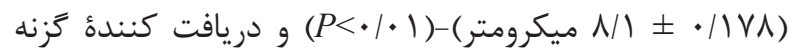

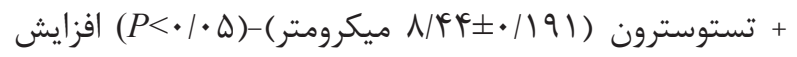

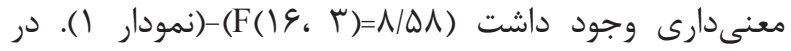

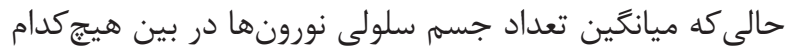
از گروهها تغيير معنى دارى را نشان نداد (نمودار كا).

$$
\text { آماده سازى حيوانات و طرح آزمايش }
$$

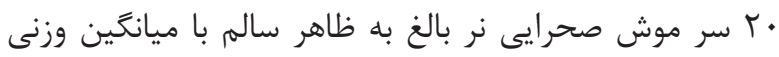

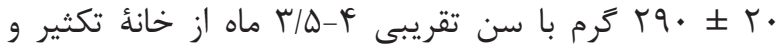

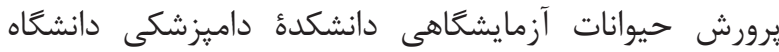

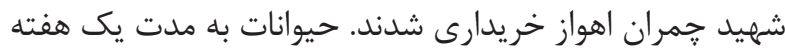

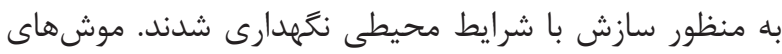

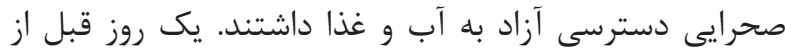

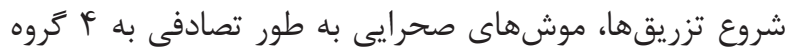

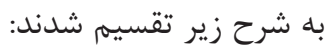

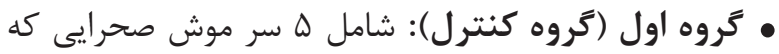

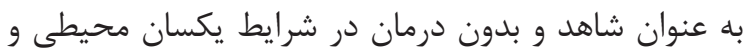
تغذيهاى با ساير كروهها بودند.

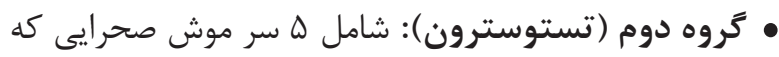

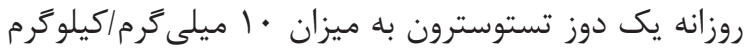
به صورت تزريق زير جلدى دريافت كردن بهند

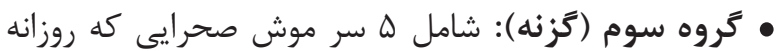

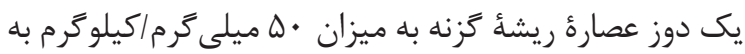

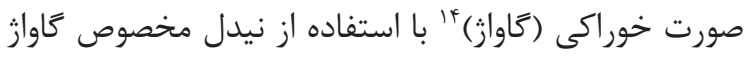

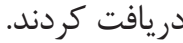

• كروه جهارم (كزنه + تستوسترون): شامل ه سر موش

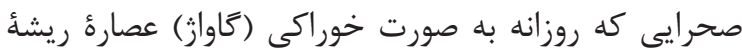

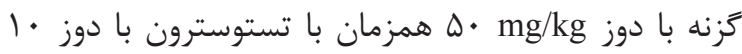

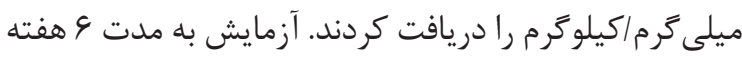
به طول انجاميد.

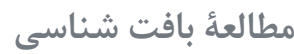
يكى روز بعد از آخرين تجويز، موشهاى صحرايى به آزمايشخاه

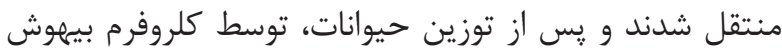

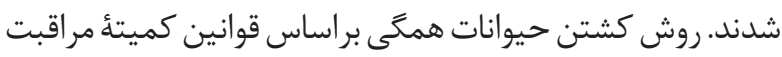

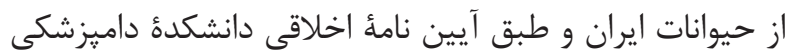

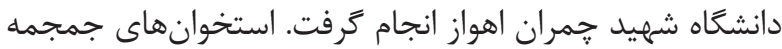

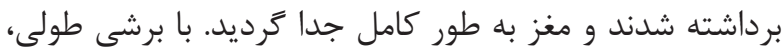

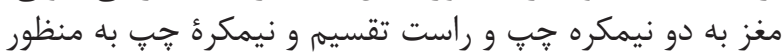

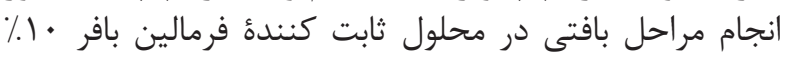

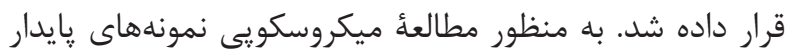

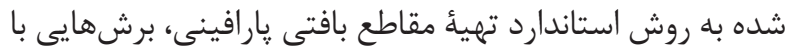

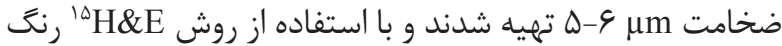
آميزى و مورد مطالعُ هيستومورفومترى قرار كرف فتند.

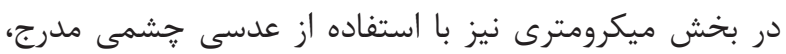

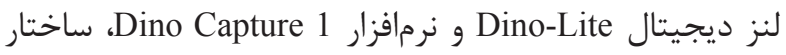

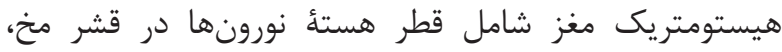
ميانكَين ضخامت لايههاى مولكولار، ترانولار و يور كنز مخجه و

\footnotetext{
${ }^{14}$ Gavage

${ }^{15}$ Hematoxylin and Eosin stain

16 Tukey
} 

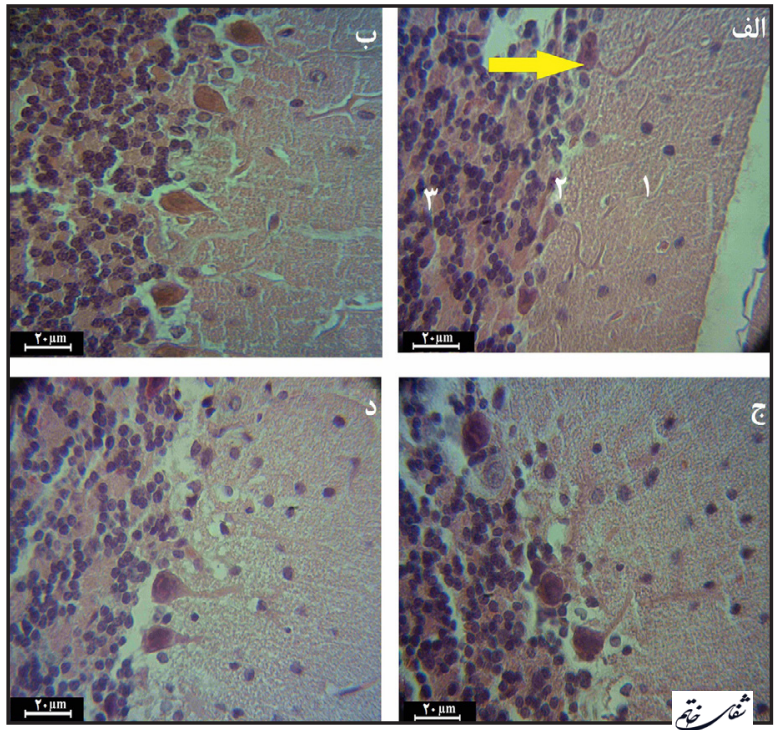

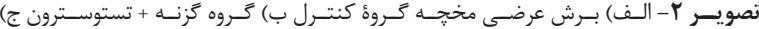

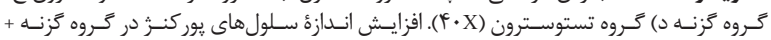

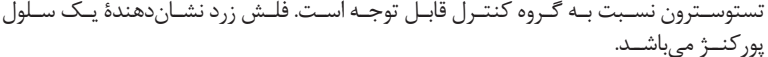

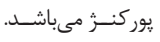

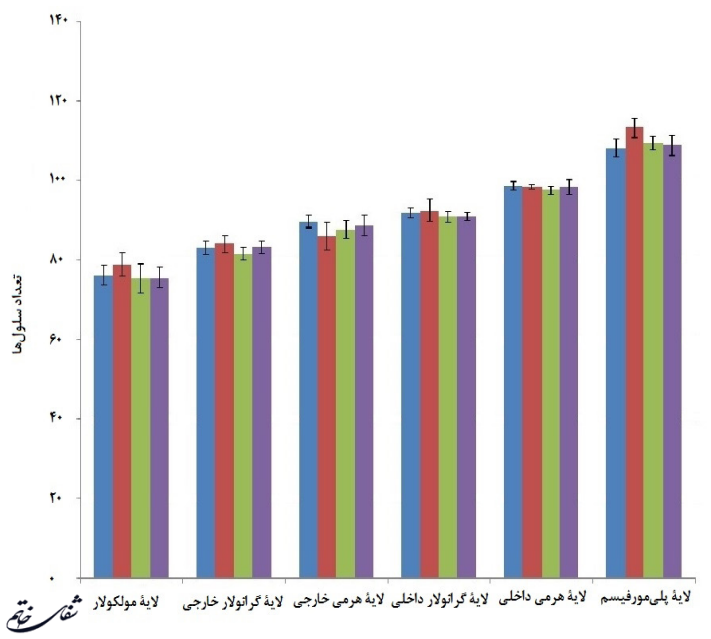

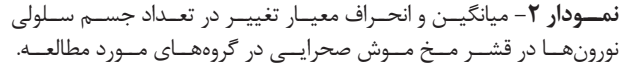

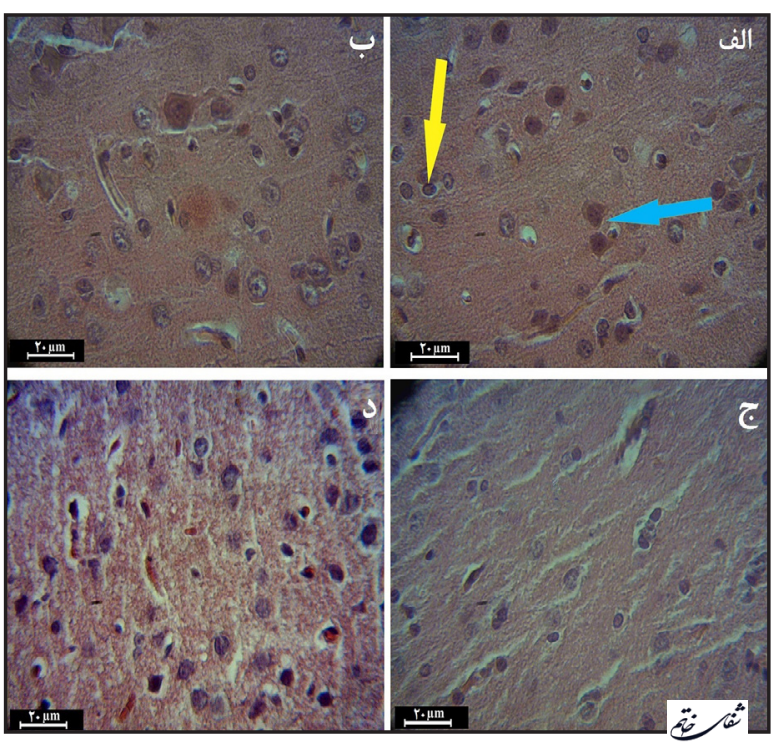

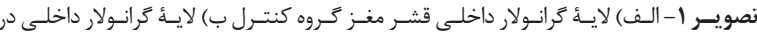

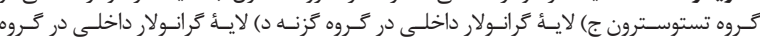

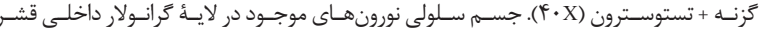

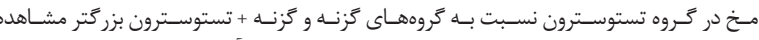

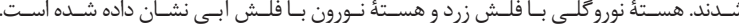

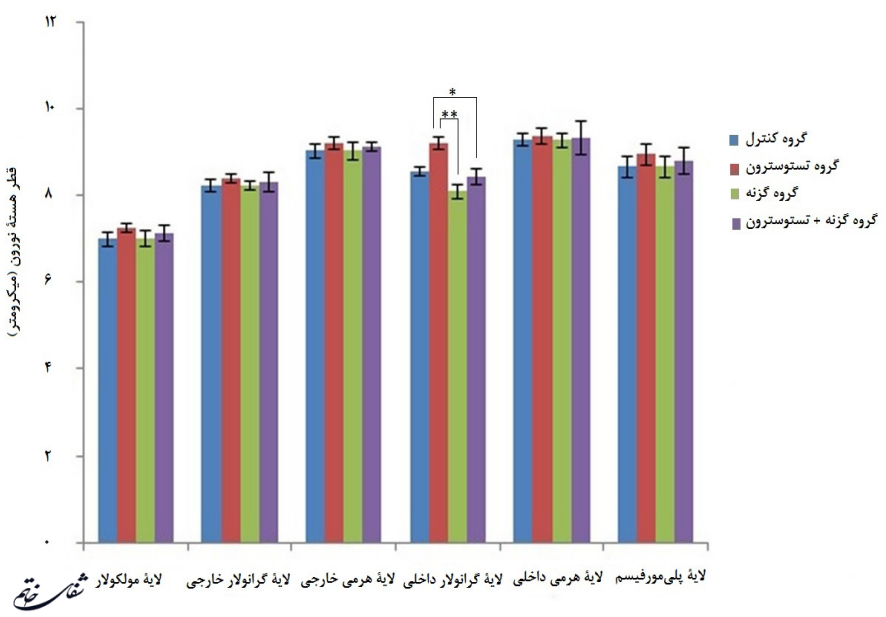

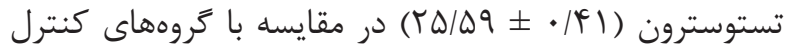

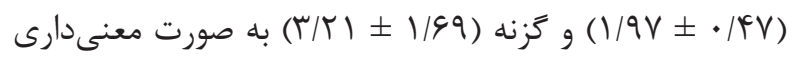

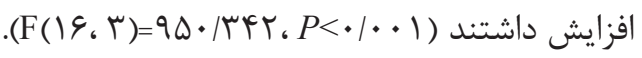

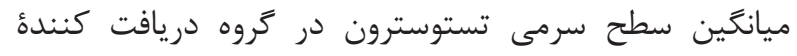

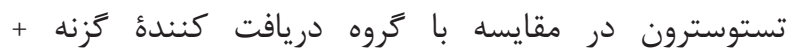

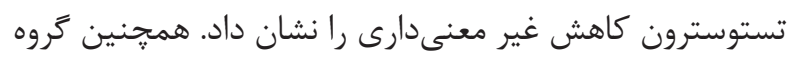

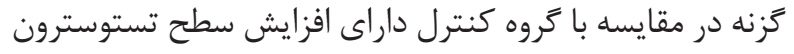
سرم خون بود ولى اين افزايش معنى دار نبود (نمودار هـ).
ميانگَين ضخامت لايههاى مولكولار، كَانولار و يور كنز در نمودار

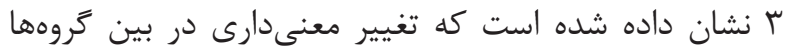

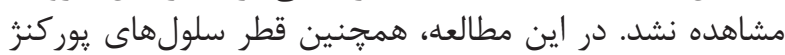

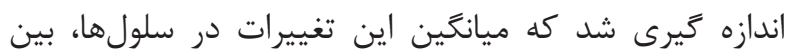
كروههاى مختلف اختلاف معنى دارى را نشان نداد (نمودار أ).

$$
\text { نتايج نمونه هاى سرمى تستوسترون }
$$

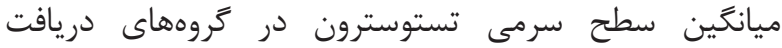

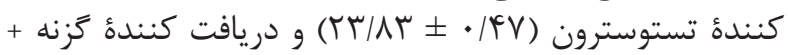




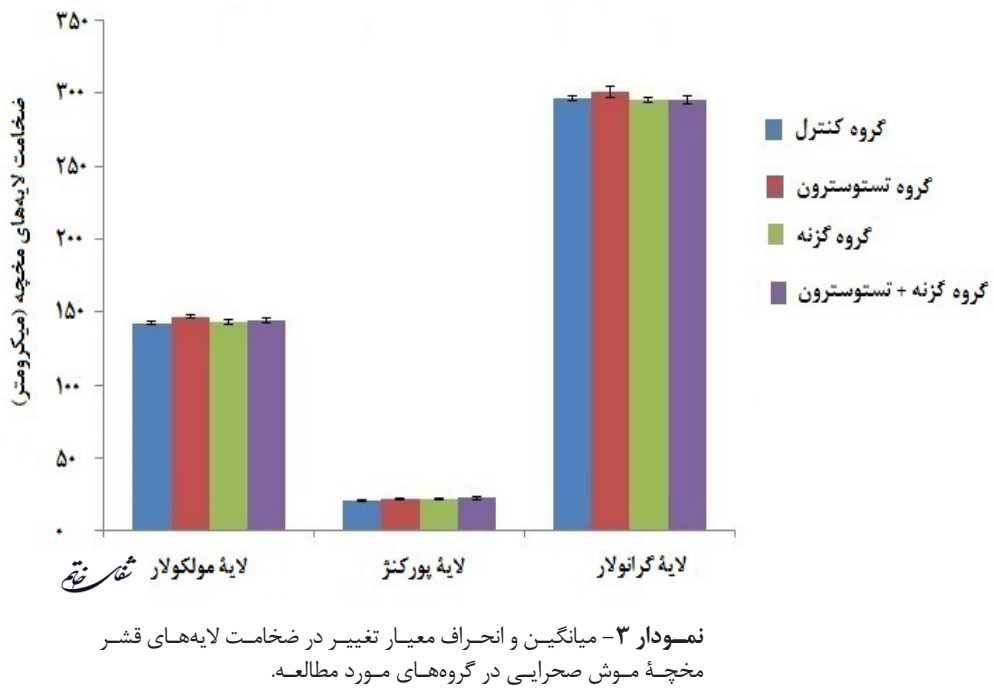

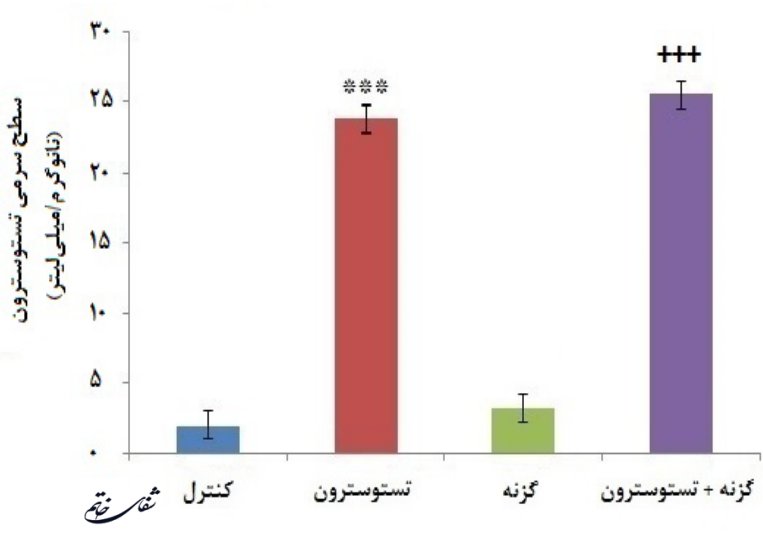

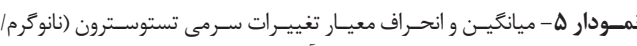

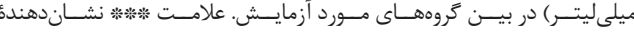

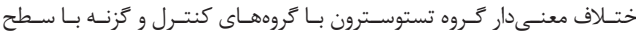

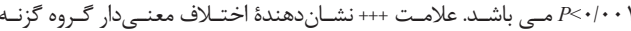

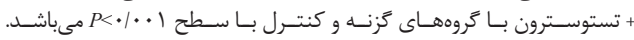

است بر روى عملكرد اين ساختارها مؤثر باشد (·) آ). نتايج حاصل

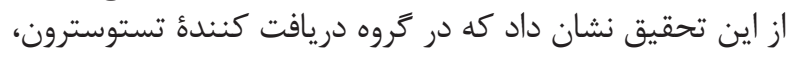

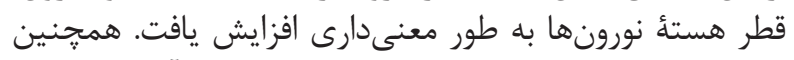

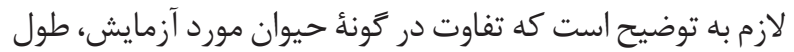

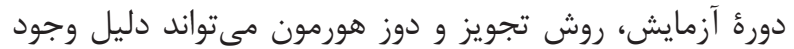

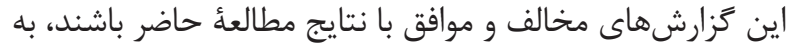

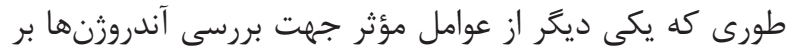

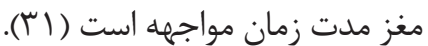

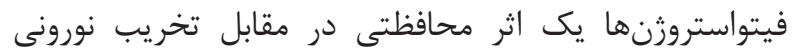

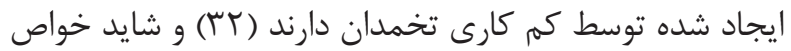

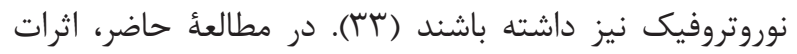

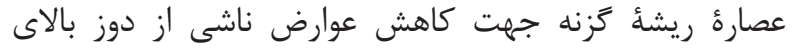

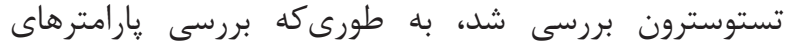

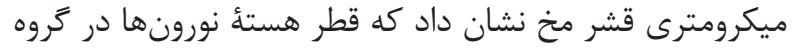

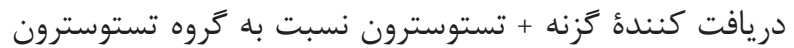

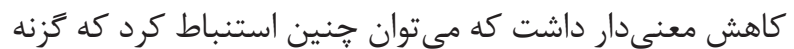
تا حدى از اثر تستوسترون جلوكيرى كردي جنه است.

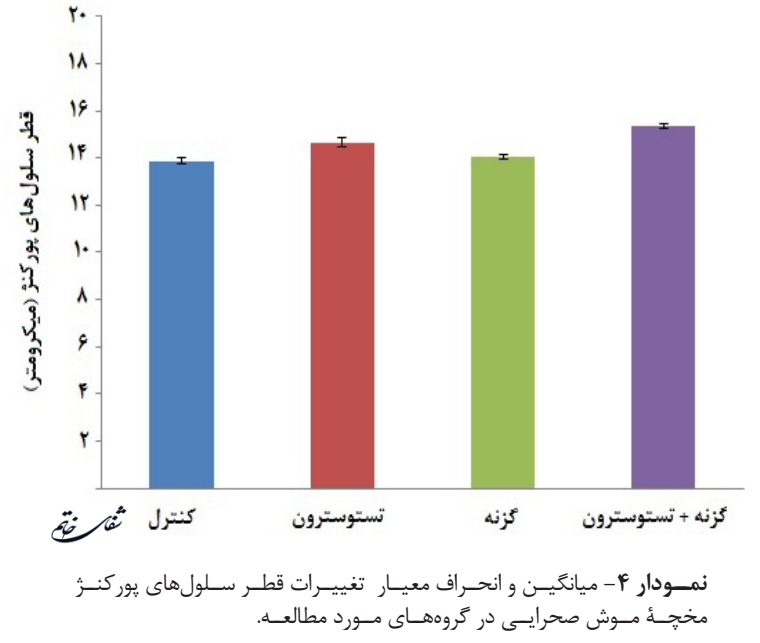

بحث و نتيجه كيرى

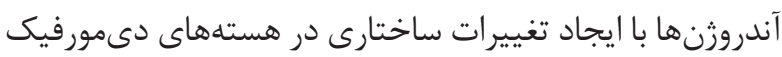

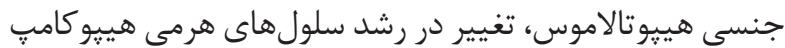

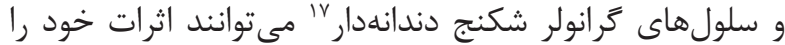

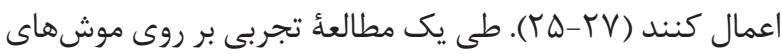

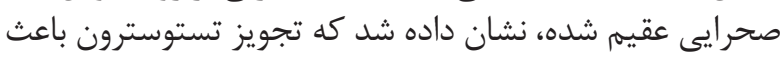

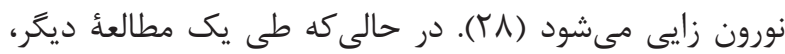
Spritzer

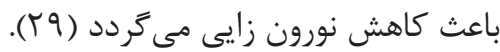
نتايج حاصل از اين تحقيق نشان داد كه در كروه تستوسترون تعداد جسم سلولى نورونها

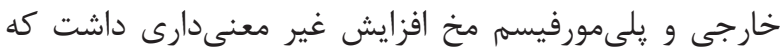

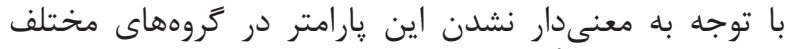

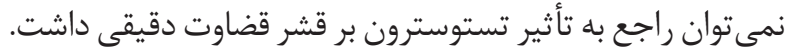

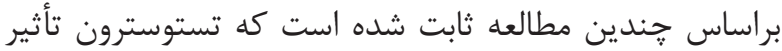

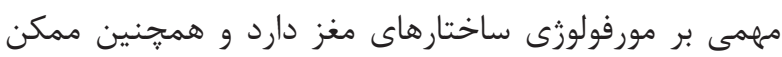




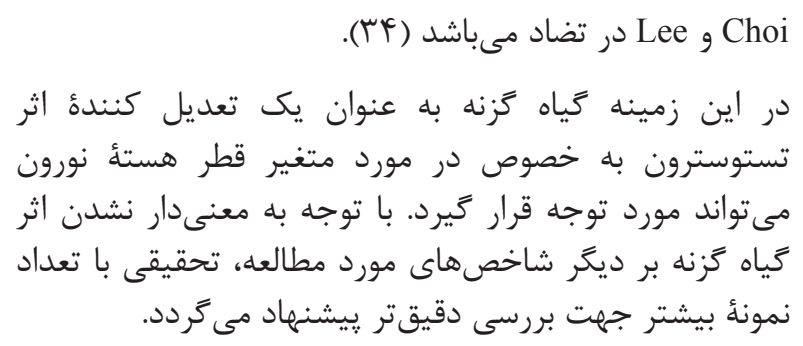

1. Stoffel-Wagner B. Neurosteroid metabolism in the human brain. Eur J Endocrinol. 2001; 145(6): 669-79.

2. Maravelias C, Dona A, Stefanidou M, Spiliopoulou C. Adverse effects of anabolic steroids in athletes: a constant threat. Toxicol Lett. 2005; 158(3): 167-75.

3. Zhang GY, Gu YQ, Wang XH, Cui YG, Bremner WJ. A Clinical Trial of Injectable Testosterone Undecanoate as a Potential Male Contraceptive in Normal Chinese Men 1. J Clin Endocrinol Metab. 1999; 84(10): 3642-7.

4. Moradi HR, Erfani Majd N, Esmaeilzadeh S, Fatemi Tabatabaei SR, editors. The histological and histometrical effects of Urtica dioica extract on rat's prostate hyperplasia. Veterinary Research Forum. 2015; 6 (1): 23-29.

5. Casey B, Duhoux S, Cohen MM. Adolescence: what do transmission, transition, and translation have to do with it? Neuron. 2010; 67(5): 749-60.

6. Giedd JN, Rapoport JL. Structural MRI of pediatric brain development: what have we learned and where are we going? Neuron. 2010; 67(5): 728-34.

7. Romer D, Walker E. Adolescent psychopathology and the developing brain : integrating brain and prevention science. Sowell ER, Thompson PM, Toga AW. Mapping adolescent brain maturation using structural magnetic resonance imaging. Adolescent psychopathology and the developing brain. Oxford University Press. 2007: 55-84.

8. Knickmeyer RC, Styner M, Short SJ, Lubach GR, Kang C, Hamer R, et al. Maturational trajectories of cortical brain development through the pubertal transition: unique species and sex differences in the monkey revealed through structural magnetic resonance imaging. Cereb Cortex. 2010; 20(5): 1053-63.

9. Cooke B. Synaptic reorganisation of the medial amygdala during puberty. J Neuroendocrinol. 2011; 23(1): 65-73.

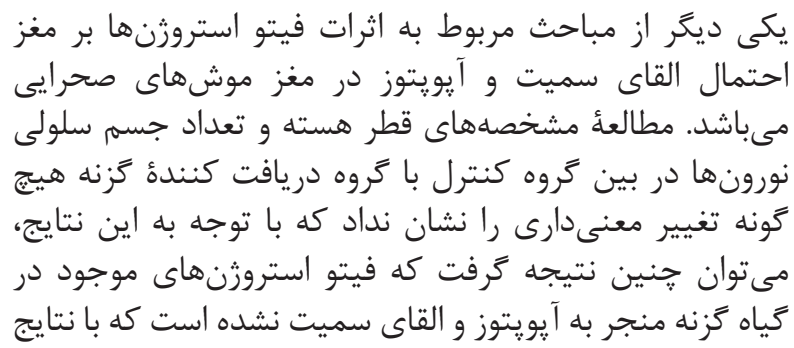

منابع

10. Lebel C, Beaulieu C. Longitudinal development of human brain wiring continues from childhood into adulthood. J Neurosci. 2011; 31(30): 10937-47.

11. Raznahan A, Lee Y, Stidd R, Long R, Greenstein D, Clasen L, et al. Longitudinally mapping the influence of sex and androgen signaling on the dynamics of human cortical maturation in adolescence. Proc Natl Acad Sci USA. 2010; 107(39): 16988-93.

12. Ho A, Villacis AJ, Svirsky SE, Foilb AR, Romeo RD. The pubertal-related decline in cellular proliferation and neurogenesis in the dentate gyrus of male rats is independent of the pubertal rise in gonadal hormones. Dev Neurobiol. 2012; 72(5): 743-52.

13. Spritzer MD, Ibler E, Inglis W, Curtis MG. Testosterone and social isolation influence adult neurogenesis in the dentate gyrus of male rats. Neuroscience. 2011; 195: 180-90.

14. Estrada M, Uhlen P, Ehrlich BE. $\mathrm{Ca}^{2+}$ oscillations induced by testosterone enhance neurite outgrowth. J Cell Sci. 2006; 119(4): 733-43.

15. Estrada M, Varshney A, Ehrlich BE. Elevated testosterone induces apoptosis in neuronal cells. J Biol Chem. 2006; 281(35): 25492-501.

16. Uchida M, Palmateer JM, Herson PS, DeVries AC, Cheng J, Hurn PD. Dose-dependent effects of androgens on outcome after focal cerebral ischemia in adult male mice. J Cereb Blood Flow Metab. 2009; 29(8): 1454-62.

17. Marty AT. The complete German commission E monographs: therapeutic guide to herbal medicines. JAMA. 1999; 281(19): 1852-3.

18. Akbay P, Basaran AA, Undeger U, Basaran N. In vitro immunomodulatory activity of flavonoid glycosides from Urtica dioica L. Pharmacognosy Res. 2003; 17(1): 34-7.

19. Schöttner M, Gansser D, Spiteller G. Lignans from 


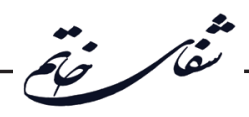

the roots of Urtica dioica and their metabolites bind to human sex hormone binding globulin (SHBG). Planta Med. 1997; 63(6): 529-32.

20. Kurzer MS, Xu X. Dietary phytoestrogens. Annu Rev Nutr. 1997; 17(1): 353-81.

21. Evans B, Griffiths K, Morton M. Inhibition of $5 \alpha$-reductase in genital skin fibroblasts and prostate tissue by dietary lignans and isoflavonoids. J Endocrinol. 1995; 147(2): 295-302.

22. Pan Y, Anthony M, Clarkson TB. Evidence for upregulation of brain-derived neurotrophic factor mRNA by soy phytoestrogens in the frontal cortex of retired breeder female rats. Neurosci Lett. 1999; 261(1-2): 17-20.

23. Jhamandas JH, Cho C, Jassar B, Harris K, MacTavish D, Easaw J. Cellular mechanisms for amyloid betaprotein activation of rat cholinergic basal forebrain neurons. J Neurophysiol. 2001; 86(3): 1312-20.

24. Adlercreutz H, Bannwart C, Wähälä K, Mäkelä T, Brunow $\mathrm{G}$, Hase $\mathrm{T}$, et al. Inhibition of human aromatase by mammalian lignans and isoflavonoid phytoestrogens. J Steroid Biochem Mol Biol. 1993; 44(2): 147-53.

25. Hine RJ, Das GD. Neuroembryogenesis in the hippocampal formation of the rat. $\mathrm{Z}$ Anat Entwicklungsgesch. 1974; 144(2): 173-86.

26. Muramatsu R, Ikegaya Y, Matsuki N, Koyama R. Neonatally born granule cells numerically dominate adult mice dentate gyrus. Neuroscience. 2007; 148(3): 593-8.

27. Morris JA, Jordan CL, Breedlove SM. Sexual differentiation of the vertebrate nervous system. Nat Neurosci. 2004; 7(10): 1034-9.

28. Spritzer MD, Galea LA. Testosterone and dihydrotestosterone, but not estradiol, enhance survival of new hippocampal neurons in adult male rats. Dev Neurobiol. 2007; 67(10): 1321-33.

29. Spritzer MD, Ibler E, Inglis W, Curtis MG. Testosterone and social isolation influence adult neurogenesis in the dentate gyrus of male rats. Neuroscience. 2011; 195: 180-90.

30. Filova B, Ostatnikova D, Celec P, Hodosy J. The effect of testosterone on the formation of brain structures. Cells Tissues Organs. 2013; 197(3): 169-77.

31. Hines M. Early androgen influences on human neural and behavioural development. Early Hum Dev. 2008; 84(12): 805-7.

32. Xu XW, Shi C, He ZQ, Ma CM, Chen WH, Shen YP, et al. Effects of phytoestrogen on mitochondrial structure and function of hippocampal CA1 region of ovariectomized rats. Cell Mol Neurobiol. 2008; 28(6): 875-86.

33. Gleason C, Cholerton B, Carlsson C, Johnson S, Asthana S. Neuroprotective effects of female sex steroids in humans: current controversies and future directions. CMLS. 2005; 62(3): 299-312.

34. Choi EJ, Lee BH. Evidence for genistein mediated cytotoxicity and apoptosis in rat brain. Life Sci. 2004; 75(4): 499-509. 